\title{
Representaciones docentes sobre las Tecnologías de la Información y Comunicación (TIC) para la enseñanza de la psicología
}

\section{Teaching representations on Information and Communication Technologies (ICT) for the teaching of psychology}

\section{Patricia Covarrubias-Papahiu}

Universidad Nacional Autónoma de México, Ciudad de México, México ORCID: http://orcid.org/0000-0001-9855-3399

Received 03-24-20 Revised 05-30-20 Accepted 07-07-20 On line 12-04-20

*Correspondence

Email: papahiu@unam.mx
Cite as:

Covarrubias-Papahiu, P (2020). Representaciones docentes sobre las Tecnologías de la Información y Comunicación (TIC) para la enseñanza de la psicología. Propósitos y Representaciones, 8(3), e428. doi: http://dx.doi.org/10.20511/pyr2020.v8n3.407 


\section{Resumen}

La adopción de las Tecnologías de la Información y la Comunicación (TIC) en la educación ha cambiado las formas de comunicación y construcción del conocimiento y producido nuevos entornos de enseñanza y aprendizaje en los últimos años, pero aunque han sido un componente indispensable de innovación curricular, no han tenido el impacto previsto en el mejoramiento de la educación, por lo que se señala la necesidad de indagar porqué las innovaciones por sí mismas no facultan a los profesores en los cambios requeridos. En tal sentido, el propósito de la investigación que se reporta fue indagar las representaciones y significados que docentes que participan en procesos de enseñanza y aprendizaje de la psicología sostienen acerca de las TIC y la experiencia que tienen al emplearlas en su práctica docente. Se realizó una investigación de tipo descriptiva e interpretativa a partir de una metodología de corte cualitativa, en la que se aplicó una entrevista semiestructurada a docentes que enseñan contenidos de psicología en instituciones de educación superior, tanto públicas como privadas, de la zona metropolitana de México. Los resultados muestran que los docentes entrevistados, en general, conciben a las TIC como innovadoras e inevitables para la práctica educativa; no obstante, y aunque le asignan diferentes significados, coinciden en que deben emplearse con ciertas restricciones. Si bien prevalece un uso pragmático e instrumental entre los docentes, consideran que las TIC facilitan los procesos de enseñanza y aprendizaje.

Palabras claves: Tecnologías de la Información y Comunicación (TIC), representaciones sociales, psicología, docentes, educación superior

\section{Summary}

The adoption of Information and Communication Technologies (ICTs) in education has changed the ways of communicating and building knowledge and produced new teaching and learning environments in recent years, but although they have been an indispensable component of curricular innovation, they have not had the expected impact on improving education. In this sense, the purpose of the research reported was to investigate the representations and meanings that teachers who participate in processes of teaching and learning psychology hold about ICTs and the experience they have in using them in their teaching practice. A descriptive and interpretative type of research was carried out based on a qualitative methodology, in which a semi-structured interview was applied to teachers who teach psychology contents in higher education institutions, both public and private, in the metropolitan area of Mexico. The results show that the teachers interviewed, in general, conceive ICTs as innovative and unavoidable for educational practice; nevertheless, and although they assign different meanings to them, they agree that they should be used with certain restrictions. Although pragmatic and instrumental use prevails among teachers, they believe that ICTs facilitate teaching and learning processes.

Keywords: Information and Communication Technologies (ICT), social representations, psychology, teachers, higher education

\section{Introducción}

Es por muchos conocido el modo en que las Tecnologías de la Información y la Comunicación (TIC) han cambiado la forma en que vivimos, nos relacionamos, trabajamos y aprendemos, y 
cómo éstas han sido consideradas como un componente ineludible en las reformas curriculares de todos los niveles educativos de México. También es reconocido el papel protagónio que tienen los docentes en materializar estas tecnologías en los contextos educativos.

En tal sentido, se consideró necesario indagar si docentes que participan de la enseñanza de la psicología incorporan las TIC en su práctica docente, y analizar si la forma en que se apropian de éstas permite superar los problemas de la enseñanza tradicional de la psicología. ${ }^{1}$

Las TIC como medio para promover la calidad de la educación y la eficiencia en el aprendizaje, han sido adoptadas por muchos países, principalmente por los argumentos que se exponen en el informe de la Organización para la Cooperación y Desarrollo Económico (OCDE, 2003), que van desde argumentos económicos, en tanto consideran la necesidad de personal competente en el uso de las TIC por el mercado laboral; argumentos sociales, que aluden a las TIC como requisito para participar en una sociedad en la que los servicios en línea son cada vez más frecuentes; hasta argumentos pedagógicos, que aseguran que las TIC favorecen el aprendizaje al desarrollar la capacidad de pensar con independencia, promueven la gestión del propio aprendizaje y la solución de problemas. En particular ha sido importante el impacto que las TIC han tenido en la educación abierta y a distancia (Pérez, 2011).

Las TIC, como señala Pérez (2011), han modificado las estructuras tradicionales inmóviles de espacio y tiempo por una graduación que permite generar modelos educativos flexibles y participativos en la formación, que favorecen el aprendizaje asincrónico, nuevas relaciones entre los actores, y una formación a lo largo de la vida.

Sin embargo, cuando el tema que se trata se refiere a innovaciones educativas y curriculares, es frecuente encontrar en los reportes de investigación al respecto, las estrategias o resistencias que los docentes adoptan para enfrentarse a éstas, y en las que se ha insistido reiteradamente que las innovaciones por sí mismas no habilitan a los profesores en los cambios necesarios, y por tanto es preciso indagar por qué no logran obtener el éxito previsto (Covarrubias \& Casarini, 2013).

La literatura especializada sobre el uso y apropiación de las TIC en las escuelas devela que no es suficiente reforzar los aspectos de tipo político (oficial e institucionales), económico, de infraestructura, de equipamiento o habilitación de dispositivos tecnológicos, para que incidan en el mejoramiento de la educación y su transformación. La tendencia dominante en la introducción de las TIC en las instituciones educativas revela que se reproducen, en general, las condiciones pedagógicas de la educación tradicional presencial avocada a la provisión de información sobre contenidos declarativos y disciplinares. El uso más frecuente de las TIC, tanto por profesores como estudiantes de los distintos niveles educativos, se relaciona más con la búsqueda y procesamiento de la información, y muy poco con la construcción del conocimiento y la colaboración. Se advierte así que cuando se emplean las TIC en la educación, predomina un uso técnico-instrumental y no un uso epistémico, es decir, no se aprovecha su potencial para el desarrollo de actividades intelectuales que conduzcan a la construcción conjunta del conocimiento (Monerero \& Pozo, 2008; Moreno, 2009; Covarrubias, 2017; Araya \& Orellana, 2018). También

\footnotetext{
${ }^{1}$ En el marco del proyecto de investigación sobre Evaluación y Planeación Educativa de la FES Iztacala-UNAM, el estudio que se reporta representa una aportación adicional a los estudios que hemos informado en relación a los factores que inciden en los procesos curriculares y educativos de la educación superior. Avances de estos resultados fueron presentados en el 3rd Annual International Symposium on "Higher Education in a Global World", en la ciudad de Atenas, Grecia, julio 2019. Así como en el X Congreso Nacional de Investigación Educativa (COMIE), en la ciudad de Acapulco (Guerrero), México, noviembre 2019.
} 
se ha notado con frecuencia que los profesores muestran menos seguridad y baja percepción de competencia frente a las TIC en comparación con los estudiantes (Díaz Barriga \& Moran, 2011).

\section{Enseñanza de la psicología.}

En lo que atañe a la psicologia, si bien su desarrollo como ciencia y profesión la han llevado a su legitimidad en la sociedad contemporánea, gracias a sus aportes al conocimiento y a la multiplicidad de problemas que atiende en sus diferentes campos de intervención, no obstante, se ha enfrentado históricamente a una serie de cuestionamientos sobre la cientificidad de sus conocimientos y la efectividad de su ejercicio profesional.

La condición pluriparadigmática de la psicología, al igual que varias ciencias sociales, ha repercutido en su enseñanza, pues al no ser una ciencia unificada, la hace muy compleja para su estudio y práctica profesional. Esto se ve reflejado en la diversidad de planes de estudio que existen para su enseñanza, en los que los estudiantes deben enfrentar una diversidad de psicologías, cada una con su particular posición epistemológica (Noriega \& Gutiérrez, 1995). Esta diversidad de epistemologías que contempla el positivismo, estructuralismo, funcionalismo, y la fenomenología, ha dado lugar a diferentes corrientes de la psicología que constituyen un collage o conglomerado teórico metodológico para su aprendizaje, en el que no existe un conjunto de leyes, principios o conceptos coherentes entre sí para explicar la realidad psicológica.

La propia diversidad epistemológica de la psicología ha promovido que su enseñanza sea predominantemente teórica con énfasis en la transmisión de su contenidos disciplinarios, preocupada más por la discusión de los fundamentos de los diferentes paradigmas que en la formación de habilidades y competencias profesionales, por lo que se ha señalado consistentemente su desfase entre la formación profesional de los estudiantes con las demandas sociales y ocupacionales de nuestro país (Preciado \& Rojas, 1989; Almeida, Guarneros, Limón \& Román, 1989; Castaño, 1989; Lara, 1989; Herrera, 1993; Harrsch, 1998; Castañeda, 1999; Covarrubias, 2003; Covarrubias \& Camarena, 2010; Zanatta \& Yurén, 2012).

Este escenario de la enseñanza de la psicología se complejiza al irrumpir las TIC en la educación, que si bien representan más que sus particularidades técnicas, una innovación en las formas de comunicación al aportar un lenguaje propio y códigos específicos orientados a producir modalidades de comunicación alternativas (hipertextos, multimedia) y nuevos entornos de aprendizaje colaborativo sin limitaciones de espacio y tiempo (Pérez, 2011), se requiere analizar si la adopción de éstas permite superar los problemas de la enseñanza tradicional de la psicología, rebasar su mero aprendizaje teórico y enciclopédico, por una enseñanza más enfocada a la construcción del conocimiento crítico y creativo y a la promoción de habilidades profesionales para la solución de problemas.

En relación al empleo de las TIC en la enseñanza de la psicología, Díaz Barriga \& Moran (2011) realizaron un diagnóstico para identificar los niveles de uso y apropiación de éstas con fines educativos a psicólogos educativos que impartían docencia teórica y práctica en semestres avanzados de la licenciatura en psicología. Encontraron que los docentes se ubicaban de manera predominante en el nivel 1 de los tres enfoques que plantea la UNESCO (2008) como estándares ligados a las competencias en el manejo de las TIC que deben poseer los docentes. Este nivel implica únicamente el manejo de nociones básicas de las TIC con fines educativos; solo algunos, directamente involucrados en el tema, alcanzaron los niveles 2 y 3 , que implican la profundización y generación del conocimiento, respectivamente. A su juicio, la institución de procedencia no ofrece las condiciones propicias para el acceso y apropiación de las TIC, ni en los espacios personales de trabajo o en las aulas. 
Por su parte, Moreno en 2009, encontró que tanto profesores como estudiantes de psicología de la Facultad de Psicología de la UNAM tenían un nivel y frecuencia de uso de las TIC muy limitado, ambos las usaban de forma superficial y pragmática, por lo que los recursos no se aprovechaban en su totalidad. Concluye que no existían las condiciones adecuadas que permitieran replanificar actividades con el objetivo de hacer propuestas educativas innovadoras por la carencia de recursos y capacitación para el uso de las TIC con fines educativos.

De aquí que nuestras interrogantes fueron ¿cómo conciben o representan las TIC en la educación docentes universitarios que enseñan contenidos de psicología?, ¿cómo representan su incorporación en los procesos de enseñanza y aprendizaje?, y ¿cuál ha sido su experiencia en su empleo? Interrogantes que parten de tener presente que el profesor es el primer mediador entre las prescripciones curriculares o innovaciones educativas y la práctica pedagógica (Young, 1981; Gimeno \& Pérez, 1989; Gimeno, 1995).

\section{Perspectiva teórica-metodológica de la investigación}

El objetivo de la investigación fue analizar las representaciones y el significado que profesores universitarios que enseñan contenidos de psicología tienen acerca de las Tecnologías de la Información y Comunicación (TIC), así como la importancia que desde su perspectiva tiene utilizar éstas en los procesos de enseñanza y aprendizaje de la psicología de los que participan, y la experiencia que tienen de su uso en la práctica educativa.

Consideramos las representaciones sociales como unidad de análisis, en tanto que en el ámbito educativo tienen gran significado ya que a partir de ellas se revelan los fenómenos que no se aprecian a simple vista, pero condicionan el proceso de enseñanza y aprendizaje.

La representación social “... es una modalidad particular del conocimiento cuya función es la elaboración de los comportamientos y la comunicación entre los individuos. ... Es un corpus organizado de conocimientos y una de las actividades psíquicas gracias a las cuales los hombres hacen tangible la realidad física y social, se integran en un grupo o en una relación cotidiana de intercambios, liberan los poderes de su imaginación" (Moscovici, 1986, pp.17-18). Moscovici deja claro que una representación corresponde a actos del pensamiento derivados de la relación de un sujeto con un objeto, en cuya relación no se da una reproducción automática sino una representación simbólica de dicho objeto.

En correspondencia, Abric (2001) señala que una representación es a la vez producto y proceso de una actividad mental en la que el individuo o grupo reconstruye la realidad que enfrenta y le atribuye una significación específica, “... toda realidad es representada, apropiada por el individuo o el grupo y reconstruida en su sistema cognitivo, integrada en su sistema de valores que depende de su historia y del contexto social e ideológico que le circunda" (p. 12), y agrega:

La representación funciona como un sistema de interpretación de la realidad que rige las relaciones de los individuos con su entorno físico y social, ya que determinará sus comportamientos o sus prácticas. Es una guía para la acción, orienta las acciones y las relaciones sociales. Es un sistema de pre-decodificación de la realidad puesto que determina un conjunto de anticipaciones y expectativas (Abric, 2001, p.13).

Por nuestro objeto de estudio, se empleó la metodología de corte cualitativa como la más idónea, con enfoque descriptivo e interpretativo, al permitirnos obtener las representaciones de los docentes en su contexto educativo, y desde una perspectiva psicosocial (Erickson, 1989). La 
investigación cualitativa es un proceso activo, sistemático y riguroso de indagación dirigida, en la cual se toman decisiones sobre lo investigable en tanto se está en el campo de estudio, sin la pretensión de alcanzar la generalidad de los datos, aún y cuando éstos puedan llegar a ser similares en contextos diferentes (Taylor \& Bogdan, 1990).

\section{Universo de estudio.}

Por ser un estudio de tipo descriptivo e interpretativo, los participantes constituyen una muestra no probabilística (Hammersley \& Atkinson, 1994), seleccionados a partir de los siguientes criterios: docentes que enseñaran psicología en el nivel de educación superior, en cualquiera de sus áreas de especialización o temas propios de la psicología, que enseñaran en instituciones públicas y privadas de la ciudad de México y zona metropolitana; además, que fueran docentes de diferentes turnos, sexo, edad y antigüedad docente, es decir, la intención fue tener una muestra lo más diversa posible que permitiera observar la incidencia de estas condiciones en el uso de las TIC; y por supuesto, tuvieran disponibilidad para la entrevista.

Participaron 34 docentes que enseñan psicología en instituciones de educación superior: 11 en universidades públicas, y 23 en universidades privadas; 13 son varones y 21 mujeres; 16 del turno matutino, 9 del vespertino y 9 de turno mixto. Con un rango muy amplio de antigüedad docente, desde uno hasta 41 años, del mismo modo, con un rango de edad amplio, de 24 a 66 años.

Las universidades públicas visitadas fueron: Instituto Politécnico Nacional (IPN-CICS UST), CICS Santo Tomás, Facultad de Psicología de la UNAM, FES Iztacala, FES Aragón, FES Zaragoza y FES Cuautitlán de la UNAM, Universidad Autónoma del Estado de México Ecatepec, Universidad Pedagógica Nacional (UPN) Tultepec y Ecatepec, y UAM Xochimilco.

Las universidades privadas visitadas fueron: Universidad de Insurgentes Tlalnepantla, Universidad del Valle de México (UVM) Chapultepec y Lago de Guadalupe, Universidad Iberoamericana Puebla, Universidad Latinoamericana (ULA), Universidad CUDEC, Justo Sierra Cien Metros, Universidad Mexicana, UNITEC, Universidad ETAC, Instituto Mexicano de Psicooncología, ICCEL, Universidad La Salle, Universidad de Cuautitlán Izcalli (UCI).

\section{Dispositivo metodológico.}

Elegimos la entrevista semiestructurada como fuente de información, porque entre sus principales características están su flexibilidad y dinamismo para obtener de forma casual, menos rígida o menos formal, las representaciones y los sentidos que los docentes asignaran a las TIC (Taylor \& Bodgan, 1990).

Las entrevistas se aplicaron de manera individual y libre durante 2016 y $2017^{2}$, a partir de un guion de preguntas orientadoras o nucleares que aseguraran explorar los ejes básicos de la investigación, las cuales se recuperan en el análisis de la información obtenida. Las entrevistas fueron grabadas y transcritas para su análisis.

El proceso analítico-interpretativo de la información obtenida contempló la búsqueda de elementos comunes en todas las entrevistas y la relación de estos elementos con conceptos o categorías teóricas pertenecientes al campo de estudio sobre la enseñanza, lo que permitió la

\footnotetext{
${ }^{2}$ Las primeras nueve entrevistas fueron aplicadas por la autora en 2016, las otras entrevistas fueron aplicadas por estudiantes del séptimo semestre de la carrera de psicología de la FES Iztacala en el periodo escolar 2017-1, quienes fueron capacitados en su metodología.
} 
construcción de categorías teórico-analíticas (Hammersley \& Atkinson, 1994). Se muestran ejemplos en la Tabla 1.

Este proceso permitió organizar y presentar la información alrededor de tres ejes principales: a) significado de las TIC para la educación, b) representaciones de las TIC para la enseñanza y aprendizaje de la psicología, c) experiencias en el uso de las TIC.

Tabla. 1

Ejemplo de categorización de la información obtenida.

¿Qué piensa usted sobre el uso de las TIC en la educación?

\begin{tabular}{|c|c|c|c|c|}
\hline $\begin{array}{l}\text { Núm. } \\
\text { Entrevista }\end{array}$ & Transcripción & $\begin{array}{l}\text { Categoría } \\
\text { social }\end{array}$ & $\begin{array}{l}\text { Categoría } \\
\text { analítica }\end{array}$ & $\begin{array}{l}\text { Categoría } \\
\text { teórica }\end{array}$ \\
\hline $\begin{array}{l}\text { (E4, profesor, } \\
\text { U. Justo Sierra, } \\
\text { p.1) }\end{array}$ & $\begin{array}{lr}\text { "Sí, r son } \\
\text { necesarias, no } \\
\text { sólo básicas sino } \\
\text { se pueden } \\
\text { inventar cosas } \\
\text { innovadoras, } \\
\text { muy necesarias } \\
\text { para estos } \\
\text { tiempos. Son } \\
\text { fundamentales y } \\
\text { hasta inevitables } \\
\text { como parte de la } \\
\text { clase" }\end{array}$ & $\begin{array}{l}\text { "Se pueden } \\
\text { inventar cosas } \\
\text { innovadoras ... } \\
\text { Son } \\
\text { fundamentales y } \\
\text { hasta inevitables } \\
\text { como parte de la } \\
\text { clase" }\end{array}$ & $\begin{array}{l}\text { Tecnología } \\
\text { obligatoria para } \\
\text { la educación }\end{array}$ & $\begin{array}{l}\text { Innovación } \\
\text { tecnológica } \\
\text { incorporada a la } \\
\text { educación }\end{array}$ \\
\hline
\end{tabular}

\section{¿Qué experiencia tiene en el empleo las TIC en su prácica docente?}

\begin{tabular}{|c|c|c|c|c|c|}
\hline $\begin{array}{l}\text { (E6, profesor, } \\
\text { UVM, p. 1) }\end{array}$ & $\begin{array}{l}\text { "Para preparar } \\
\text { una clase, por } \\
\text { ejemplo, } \\
\text { investigo el tema } \\
\text { y busco por } \\
\text { ejemplo en } \\
\text { Youtube, porque } \\
\text { hay un montón de } \\
\text { tutoriales en } \\
\text { educación y } \\
\text { luego ya lo } \\
\text { integro a la } \\
\text { presentación o al } \\
\text { prezi, } \\
\text { dependiendo" }\end{array}$ & $\begin{array}{lr}\text { "... investigo el } \\
\text { tema y busco por } \\
\text { ejemplo } \\
\text { Youtube } & \text {... } \\
\text { luego y } & \text { ya lo } \\
\text { integro a la } \\
\text { presentación o al } \\
\text { prezi" }\end{array}$ & $\begin{array}{l}\text { Recursos } \\
\text { visuales } \\
\text { audiovisuales c } \\
\text { la WEB }\end{array}$ & & $\begin{array}{l}\text { Recursos } \\
\text { digitales para el } \\
\text { aprendizaje } \\
\text { (RDA) }\end{array}$ \\
\hline
\end{tabular}

Fuente: Elaboración propia

\section{Resultados}


Se presentan las categorías obtenidas en cada uno de los ejes construidos. Por cuestiones de espacio se reuperan solo las tendencias generales encontradas, y se presentan solo algunos de los discursos más representativos de los docentes entrevistados, que sustentan las interpretaciones realizadas.

\section{Significado de las TIC para la educación.}

Ante la pregunta ¿Qué piensa usted sobre el uso de las TIC en la educación?, en general los docentes entrevistados consideran relevante y positivo el uso de las TIC en la educación, aunque les asignan diferentes significados. Apreciamos así, que si bien la mayoría piensa que es innegable su potencial innovador en la educación, diversos profesores piensan que conllevan riesgos y su empleo requiere determinadas restricciones.

\section{Son innovadoras $e$ inevitables.}

Desde la perspectiva de la mayoría de los profesores, el uso de las TIC no solo es indispensable e ineludible para la educación acorde a la época actual, sino que representa la posibilidad de innovar durante la práctica educativa. Exponemos solo dos de sus discursos:

Sí, son necesarias, no sólo básicas sino se pueden inventar cosas innovadoras, muy necesarias para estos tiempos. Son fundamentales y hasta inevitables como parte de la clase (Entrevista 4, Profesor U. Justo Sierra, p. 1)

Al ser tecnología, la escuela necesita estar a la vanguardia y estar actualizándose en cuanto a contenido y medio para impartir clases de calidad a los alumnos (E33, profesora, UVM Lago de Gpe., p. 1)

Los docentes priorizan la innovación por sí misma como elemento indispensable para incorporar las TIC a la educación, sin que analicen su importancia o pertinencia en el proceso educativo. Esta representación coincide con lo encontrado en diversos estudios que han analizado las actitudes de los docentes frente a las TIC. García-Valcárcel (2003, cit. por Riascos-Erazo, Quintero-Calvache \& Ávila-Fajardo, 2009) al analizar estos estudios encontró que una de las actitudes básicas entre los docentes es considerar a las TIC como "imprescindibles" para la educación, pero del mismo modo, los docentes destacan su innovación sin analizar su importancia o incidencia en el proceso de enseñanza y aprendizaje, o el impacto que tiene su incorporación en el currículum.

Parecería entonces que la innovación como tal, o tal vez como un rasgo distintivo que caracteriza a la sociedad contemporánea, pasa a ser parte de la representación social colectiva, por lo que se piensa que debe expresarse en todos los ámbitos de la vida, incluyendo la educación, y por tanto no se espera se cuestione su pertinencia o adecuación en ésta.

\section{Promueven la distracción ... se pierde la concentración.}

Sin embargo, aunque se valora a las TIC como necesarias para la innovación educativa en los tiempos actuales, varios docentes también perciben que promueven el descuido y la distracción en los estudiantes:

[...] aportan innovación en el aula, solo que a veces los alumnos las utilizan como distractores, como el caso del uso del internet y pierden la concentración en la clase, yo creo que son eficaces para enseñar de mejor forma, pero debería haber un mayor control de ellas (E5, profesora, U. Insurgentes, p. 1). 
Pues, a decir verdad, pienso que son una navaja de doble filo. Realmente si se utilizan para lo que están destinadas, son herramientas fabulosas, pero si no se tiene control sobre ellas podría ser que estén generando distracciones que no enriquezcan el aprovechamiento educativo del alumno (E18, profesor, Instituto Mexicano de Psicooncología, p. 1).

\section{Generan expectativas utópicas: promueven la flojera, simulación, la falta de ética y su dependencia.}

Muy relacionada con la representación anterior, también hay quienes consideran que es una idea ilusoria pensar que las TIC sirvan para promover que los estudiantes trabajen con calidad y y competencia, siendo que favorecen más el que los estudiantes no se comprometan en su aprendizaje al prestarse para la simulación, el plagio, el comportamiento poco ético, o la dependencia a éstas:

Pues creo que una expectativa más utópica es que las TIC se utilicen para realizar trabajos de calidad, con profesionalismo y que no sirvan para haraganería que es para lo que muchas veces los alumnos las usan [...] pueden llegar a ser eficientes pero también pueden prestarse para un mal uso, me ha tocado muchas veces revisar trabajos en los que hay enlaces directos de cosas que los alumnos encuentran en el rincón del vago, en wikipedia ... las tecnologías para empezar deben enseñarles bien a los alumnos a usarlas, a usarlas con profesionalismo, con ética para que no se den ese tipo de situaciones (E9, profesor, Universidad Mexicana, p. 1).

Estaría a favor siempre y cuando el uso sea el adecuado sin abusar de ese tipo de instrumentos (E14, profesora, U. Insurgentes, p. 1)

Vemos entonces que, aunque los docentes consideran que las TIC pueden llegar a eficiente la enseñanza y el trabajo de los estudiantes, también piensan que pueden suscitar un pobre desempeño académico.

Estas representaciones coinciden con lo encontrado por Moreno (2009) y Pérez (2011), quienes observan que, si bien el internet o "la Web" se ha convertido en uno de los recursos de aprendizaje y enseñanza disponibles tanto para profesores como estudiantes, lo más frecuente es encontrar que ambos lo usan con fines distintos a los académicos.

También se cuenta con investigaciones que advierten que los estudiantes, con todo y que buscan información relevante en internet, así lo usan para obtener música, comunicarse por "chat", inclusive usan Facebook mayormente para estar en contacto con amigos que con fines académicos (Abdulsalam \& Azizah, 2013).

La accesibilidad a todo tipo de información de forma inmediata conlleva ventajas, pero también riesgos. De aquí que los docentes argumenten que se requiere de control, restricciones, profesionalismo y ética para que el uso de las TIC en la educación sea eficiente.

\section{Conflictivas, quitan tiempo.}

Aunque menos frecuente, también hay profesores que piensan que su uso es incorrecto a tal grado que llegan a ser "conflictivas", de difícil manejo: 
[sobre las TIC] conflictivas, no me gusta meterme con ellas [...] Son conflictivas en sentido de que no hay una organización para emplearse correctamente, y te voy a dar un ejemplo bastante vago. Verás, en mis grupos suelen hacer siempre grupos de Facebook por materia o grupo y casi siempre funciona como una especie de teléfono descompuesto. Porque se trata de un constante "El profesor me dijo que..." "escuché que el profe quiere que..." y realmente la información se pierde muy cañón (E30, profesor Fac. Psicología-UNAM, p. 3).

no me gustan las aulas virtuales me parece que me quitan mucho tiempo y es demasiado tiempo invertido para lo que aportan desde mi perspectiva y mi experiencia, pero pues hay profesores que están encantados con su aula virtual (E27, profesora FES ZaragozaUNAM, p.1).

Para estos docentes, las TIC representan problemas e inversión de tiempo poco productivo al considerarlas solo como medios de comunicación con sus estudiantes, o medios de contacto con fines informativos, más que como herramientas tecnológicas con propósitos educativos.

\section{Representaciones de las TIC para la enseñanza y aprendizaje de la psicología.}

Ante la pregunta ¿Qué importancia tiene para usted el empleo de las TIC para la enseñanza de los contenidos que usted imparte?, o bien, ¿Cómo considera que incide el empleo de las TIC en el aprendizaje de los estudiantes?, si bien encontramos diversidad de representaciones, en su gran mayoría atribuyen un uso positivo de las TIC en el proceso educativo, que van desde considerar que su empleo permite captar la atención de los estudiantes, mejorar la comprensión de los contenidos, y/o facilitar su vínculo con los estudiantes, hasta quienes consideran que sin su empleo se dificulta el aprendizaje. No obstante, también hay pronunciamientos en contra.

\section{Hacen más dinámicas las clases y promueven la atención de los alumnos.}

Una idea frecuente entre los docentes es que las TIC son una herramienta fundamental para hacer las clases más dinámicas y captar la atención de los estudiantes, en tanto proporcionan recursos digitales y medios audiovisuales que facilitan la enseñanza, permiten presentar información adicional y proporcionar ejemplos:

si por ejemplo, si son temas muy teóricos muchas veces las TIC nos ayudan a que los chicos no se duerman, no se aburran, les llame la atención. Pero cuando se requieren de temas más prácticos es indispensable sacarlos del aula, poder hacer la práctica en contextos reales más que en contextos digitales (E14, profesora, Universidad Insurgente, p.2).

Es un apoyo grande ya que hace dinámicas las clases y me permite dar los contenidos de forma más clara [...] el que no puedas utilizar estas herramientas dificulta la dinámica del salón, la atención de los muchachos, así como el aprendizaje que se llevan, ya que al momento de evaluar puedes darte cuenta que pusieron atención cuando te dicen "Sí miss, de hecho lo puso en el vídeo de las adicciones" y allí te das cuenta que el apoyo visual ayuda bastante en el proceso de enseñanza - aprendizaje (E33, profesora UVM Lago de Gpe., p. 1). 


\section{Los temas se comprenden mejor.}

Para otros, las TIC facilitan la comprensión de los temas durante la enseñanza, aunque nuevamente esta comprensión se facilita por la accesibilidad a la información y a materiales audiovisuales que la tecnología posibilita:

Son fundamental para dar una mejor clase y que sea más funcional $[\ldots]$ quiero decir que los temas se comprendan mejor, que los alumnos tengan otra visión y en el caso de buscar temas, que se hagan mejores búsquedas de información para sus investigaciones (E5, profesora, U. Insurgentes, p.1).

Facilitan la comprensión, es básico en la actualidad que lo acompañemos con algo visual e interactivo (E31, profesora, Iberoamericana Puebla, p. 1).

\section{Responden a las necesidades de los estudiantes.}

Otra idea entre varios docentes es que el empleo de las TIC en la enseñanza es inexorable dada la familiaridad que los estudiantes tienen con ellas y lo que representan en su vida diaria:

Sí, por supuesto, sucede que los estudiantes, en los distintos niveles educativos, pero sobre todo a nivel licenciatura, tienen ya un uso bastante avanzado sobre estas tecnologías y si desde la docencia no las implementamos, los que nos vamos rezagando somos nosotros, es decir, lo que podamos ofrecer puede quedarse como limitado o poco atractivo respecto a una herramienta que ellos ya traen (E1, profesora, Justo Sierra Cien Metros, p. 1)

Si ellos las usan, entonces a veces uno como profesor dices, 'bueno yo no quiero tener un face.. pero finalmente es el propio alumno el que se comunica por vía face...' y en algunas ocasiones ellos terminan enseñándome (E22, profesora, Universidad de Cuautitlan Izccalli, p.1).

\section{Facilitan la enseñanza y el vínculo profesor-alumno.}

Para otros docentes, las TIC además de que les facilitan la enseñanza, representan la oportunidad de establecer vínculos con sus estudiantes, gracias a que éstas posibilitan el acceso a todo tipo de contenidos de la psicología:

Nos han facilitado la enseñanza y el vínculo que podemos establecer con los alumnos, por lo que no le veo el visto malo de que las utilicemos, siempre y cuando sea con responsabilidad de lo que subimos y lo que bajamos de internet [...] Hasta ahora no me he encontrado con algún tema psicológico que no esté en internet (E34, profesor Universidad Cuautitlán Izcali, p. 1).

Tengo más contacto directo con los chicos porque si tienen alguna duda con el material pues ya me escriben y ya les contesto, ... a veces la verdad el material está muy difícil y busco información adicional, ya sean artículos o audiovisual para que sea más comprensivo el tema, y sin esta herramienta tan funcional no podría facilitar todo esto, tendríamos que pasar horas en la biblioteca para encontrar materiales y si hay duda esperar hasta que nos veamos la siguiente clase y esto atrasaría el semestre (E24, profesora, CICS Sto. Tomás, p.2). 
Las representaciones hasta aquí analizadas connotan una aceptación amplia y positiva de las TIC por los docentes al considerar que son un apoyo a la enseñanza que permiten desarrollar clases más atractivas, más dinámicas, más "visuales" e interactivas, facilitan la comprensión de los temas, permiten la comunicación constante con los estudiantes, en fin, hacen "más funcional" las clases; no obstante, lo atribuyen a que éstas suministran todo tipo información, "captan la atención" de los estudiantes, hacen más amena la instrucción y están en sintonía o son inherentes al mundo de los jóvenes. Vemos entonces que hay una idea frecuente de tipo pragmático e instrumental de las TIC entre los docentes, cuyos recursos facilitan sus actividades de enseñanza, particularmente la búsqueda y transmisión de información, pero sin que haya una representación de éstas vinculadas al desarrollo de competencias cognitivas en torno a aprendizajes significativos y construcción del conocimiento.

\section{No sustituyen los métodos de enseñanza que los docentes han construidos.}

No obstante, para algunos docentes, las TIC, si bien son útiles, esto no significa que reemplacen los métodos de enseñanza construidos por los docentes a través de su experiencia, ni los suplen en su función docente, solo los complementan:

Son muy útiles si se piensan como un medio de complementar lo visto en clases y no como algo que "sustituya" los métodos de enseñanza ya establecidos, no sé si me explico, mira cada profesor tiene una forma de enseñar que se va construyendo con base a sus experiencias y conocimientos (E29, profesora IPN-CICS UST, pp. 1-2)

... algo muy real y es que las TIC no sustituyen el contenido, planeación, experiencia docente, por muy colorido, muy impactante, por supuesto que no sustituye, que la base siempre va a hacer la planeación, toda la parte de carta descriptiva que hace el docente, eso es básico (E10, profesora UNITEC/ULA, p.2).

En estos testimonios se atribuye al docente un papel protagónico en el proceso educativo, cuya experiencia y conocimientos determinan la calidad de la enseñanza, y las TIC se conciben solo como recursos complementarios que auxilian al docente a mantener la atención o concentración de los estudiantes.

\section{No mejoran por sí mismas el aprendizaje.}

Muy vinculada con la representación anterior, para algunos docentes, son los estudiantes y profesores los que construyen conjuntamente el conocimiento, donde las TIC nuevamente solo cumplen una función de apoyo a la docencia:

Depende de la clase, tal vez en unas clases pues si se presta, no sé, hay veces una simple presentación, pues hay veces que si beneficia para hacer más claro un tema, ya sea con ayuda de imágenes o videos, permite que el alumno pueda comprender un poco más, pero no creo que por sí mismas hagan mejor la clase, no sé si me explique, o sea, la clase es buena porque los alumnos y el profesor la construyen así, y eso es adicional, puede ser como un plus que puede ayudar a la clase, pero pues no lo es todo [...] entonces insisto, las TIC son una herramienta, no son el aprendizaje (E27, profesora FES Zaragoza-UNAM, p.2).

bajo este esquema, quien no cumple con actividades o no desarrolla habilidades para ser autodidacta no podrá concluir sus estudios. Las usarán en mayor medida personas responsables y que se hacen cargo de su propio conocimiento (E12, profesor, U. Mexicana y ETAC, p. 2). 
Aquí se atribuye tanto al docente y como al estudiante un papel activo en el proceso educativo, pero también en el propio uso de las TIC. Como señalara Coll (2008), si bien las TIC llegan a ser instrumentos potenciales para promover el aprendizaje, no son las TIC por si misma sino las actividades que llevan a cabo profesores y estudiantes con ellas donde se encuentran los indicadores para valorar impacto en los procesos de enseñanza y aprendizaje.

\section{Fomentan el copiar y pegar.}

Para otros profesores las TIC no solo no garantizan el aprendizaje, sino que pueden llegar a entorpecerlo:

en mi caso no me funcionó mucho utilizarlas porque fomentó más el copiar y pegar y dejaban de lado cosas importantes (E9, profesor, Universidad Mexicana, p. 2).

Pienso que a veces no se les explota de la manera correcta o no se les da el uso que debe de ser, hablando en términos académicos; para la calidad de los trabajos o de las presentaciones se necesitan programas específicos e incluso a veces entregan unos trabajos muy "mediocres" los chicos y aun así los tengo que pasar (E33, profesora, UVM Lago de Gpe., p. 2).

El problema para estos profesores es que, si bien las TIC son recursos valiosos, también se prestan para un uso inadecuado pues el acceso a todo tipo de información no asegura que los estudiantes seleccionen los materiales adecuados y realicen trabajos de calidad, es frecuente encontrar estudiantes que descargan información de internet sin que la analicen. Este problema ya ha sido reportado por investigadores que han indagado su uso en estudiantes de psicología. Moreno (2009), por ejemplo, encontró que, en tareas escolares, los estudiantes generalmente hacen una división en equipo y usan las TIC solamente para unir archivos, o se asignan la transcripción, mientras que otros recopilan información o alguna otra tarea relacionada.

Por su parte, Romero \& Hernández (2011) encontraron que la percepción que tienen estudiantes de psicología sobre sus propias habilidades tecnológicas en el uso de las TIC son de nivel básico, en tanto y en palabras de algunos "Me gusta más recurrir a internet porque hay veces que me encuentro páginas interesantes, y para empezar me llaman la atención las imágenes, cómo está estructurada la información, y ya empiezo a leer y ... copiar y pegar” (p. 41); “... es un medio muy fácil para ponerte de acuerdo y quizás desde ahí mismo unir los trabajos, o buscar más información" (p. 43). Los autores concluyen, que la realización de tareas por los estudiantes a partir de la información hallada en internet, suelen ser reproductivas e irreflexivas, por lo que podrían juzgarse de "bajo nivel cognitivo" y de trabajo individual, sin que se aprovechen sus potencialidades para el andamiaje, la ampliación de las habilidades cognitivas y el trabajo colaborativo para la construcción conjunta del conocimiento.

\section{Las TIC no son propias para la enseñanza y aprendizaje de la psicología.}

Todavía hay docentes que piensan que las TIC no son necesarias para la enseñanza de la psicología, y lo adjudican a sus contenidos y a las formas de aprenderlos:

Supongo que en algunas cuestiones si, ya que para algunas carreras el material está al pie del cañón para que lo usen, y si necesitan más cosas, pero en psicología no, eso no es tan promovido aquí, creo que es debido a la diversidad de formas que tenemos nosotros para aprender, ya sabes, el ingenio [...] considero que también atrofian 
algunas de sus capacidades (de los estudiantes) porque dependen de ellas (E23, profesor, FES Zaragoza, p. 1).

No las uso y los foros de discusión los hago esporádicamente ya que trabajo de manera activa con los alumnos en clase [...] Yo estoy completamente a favor de cualquier herramienta que enriquezca el aprendizaje, pero creo que depende del tipo de carrera, materia y de los contenidos. Cabe aclarar que por el tipo de materias que imparto, el trabajo es presencial y de trabajo directo en instituciones con beneficiarios (E24, profesora, U. Iberoamericana, p. 2).

En estos relatos se devela resistencia de los docentes a cambiar o diversificar sus prácticas habituales de enseñanza, al argumentar que son los contenidos y los objetivos de sus cursos los que no lo permiten, sin que se aluda a las potencialidades de las TIC para diseñar escenarios educativos diferentes, o como formas alternas de construir el conocimiento en psicología. Tampoco se alude a sus potencialidades para motivar o estimular a los estudiantes. Contrasta además con lo encontrado por Covarrubias (2017) en el sentido que el tipo de disciplina y materias impartidas por docentes de diversas carreras de universidades públicas y privadas no tienen relación o algún impacto significativo con respecto a la aceptación y uso de las TIC en su práctica educativa.

\section{Experiencias en el uso de las TIC.}

Ante la pregunta ¿Qué experiencia tiene en el empleo de las TIC en su práctica docente?, de forma recurrente los docentes reconocieron tener solo conocimientos básicos para el uso de recursos digitales en sus cursos, no obstante, algunos profesores, mayormente los que imparten cursos en línea, utilizan recursos más completos e interactivos y producen materiales para apoyar el aprendizaje de sus estudiantes.

\section{De Youtube al PowerPoint.}

La experiencia más frecuente reportada por los profesores con las TIC se remite al uso de recursos digitales disponibles, de fácil acceso, es dedcir, que no van más allá de la paquetería informática de uso común:

Para preparar una clase, por ejemplo, investigo el tema y busco por ejemplo en Youtube, porque hay un montón de tutoriales en educación y luego ya lo integro a la presentación o al prezi, dependiendo (E6, profesor, UVM, p. 1).

Yo acostumbro dar clases apoyado en presentación de Powerpoint, y entonces bueno en algunas ocasiones pues muchas veces me solicitan eso y sobre eso pues me mandan dudas y esas cosas por el estilo (E11, F. de Psicología, p. 1).

\section{Uso del proyector, búsqueda en internet y redes sociales.}

También de manera consistente los docentes reportan hacer uso de imágenes, videos, esquemas, el uso de la WEB (World Wide Web), y algunas redes sociales en su práctica docente:

$\mathrm{Si}$, en algunas ocasiones, llego a utilizar el cañon para realizar presentaciones de lo que se estará revisando en la clase y hago que mis alumnos busquen documentos en internet y el uso de alguna plataforma (Entrevista 2, profesora, ULA, p. 2). 
En las materias que doy en modalidad presencial me apoyo de materiales multimedia, como videos documentales que se pueden revisar en Youtube, también genero grupos escolares de Facebook para que se hagan entregas o subir materiales que los alumnos deben revisar previamente para la clase, así como solicitar investigaciones en sitios de revistas electrónicas que tienen contenido especializado (E12, profesor, U. Mexicana y ETAC, p. 2).

La tendencia general encontrada entre los docentes es el uso de las redes sociales como Facebook, Twitter, Youtube, empleadas principalmente para compartir archivos, descargar información, descargar videos o para comunicaciones habituales. Muy pocos profesores elaboran sus propios materiales de apoyo, escasamente reportaron utilizar salas de conferencias, laboratorios de cómputo, foros de discusión, plataformas interactivas, menos aún, el diseño de ambientes de aprendizaje en línea.

Centrar el uso de las TIC en lo audiovisual y como formas de comunicación con los estudiantes también se ha encontrado en otras investigaciones (Moreno, 2009; Díaz Barriga y Moran, 2011; Echeverria, 2014), cuyo uso más frecuente por los profesores son el correo electrónico, powerpoint, internet, videos, que requieren solo nociones básicas digitales, que por si mismas no permiten la profundización y construcción del concimiento

\section{Uso de lecciones interactivas.}

El uso estratégico de las TIC para promover aprendizajes significativos en los estudiantes está prácticamente ausente entre los docentes, solo en casos excepcionales, particularmente docentes que participan en cursos en línea, conocen y emplean recursos que trascienden el manejo de nociones básicas de las TIC:

... hay una función, aplicación que utilizamos sobre todo en las materias online, que se llama Collaborative, es fantástica porque es como grabar una clase en línea, trabajo con la presentación y si conecto la cámara, mis alumnos observan en línea, en tiempo real mis alumnos hacen preguntas, comentarios, en ese momento hacemos discusión y resulta fantástico (E10, profesora, UNITEC/ULA, p. 2).

El empleo de un software de colaboración reúne una serie de programas tecnológicos que sirven de apoyo para el trabajo grupal intencional en línea, permite compartir, procesar y administrar archivos, documentos y otros tipos de datos entre varios usuarios, y dependerá del docente que la emplee brindar a cada participante las mismas oportunidades para participar y comunicar sus ideas y experiencias, como un medio que permita la creación conjunta de saberes, utilizando lo virtual para la construcción del conocimiento (Salinas, 2008).

García-Valcácer, Hernández \& Recamán (2012), en su estudio sobre las opiniones de profesores y estudiantes que participaron en una experiencia de aprendizaje colaborativo a través de las TIC, encontraron que, en general, ambos atribuyeron una valoración positiva y favorable a esta metodología. Entre sus virtudes señalaron la posibilidad de que los estudiantes desarrollen autonomía crítica e interacción con los otros, lo que mejora sus capacidades sociales además de compartir y generar conocimiento, no obstante, también señalaron como desfavorable el mayor tiempo de preparación y seguimiento que implica esta metodología. 


\section{Reducen costos, tiempo y distancia.}

Para algunos profesores, el uso de las TIC representa reducción de costos de diversa índole, desde ahorro en tiempo y dinero, hasta la mitigación del impacto ambiental:

Al utilizarlas (las TIC) la información se vuelve portable y fácil de revisar prácticamente en cualquier lugar donde el alumno tenga acceso a internet, así que el problema de las entregas y los tiempos de revisar la información se reducen bastante. El beneficio es la reducción de costos, tiempos, distancias, también tiene su beneficio en cuanto al impacto ambiental (E12, profesor, U. Mexicana y ETAC, p. 2).

La manera en que puedes manejarlos, la flexibilidad que como profesor puedes adquirir, la comunicación con ellos y también el poder hacer que ahorren para que no saquen las copias y tengan el material en su celular (E32, profesora, Universidad Insurgentes Tlalnepantla, p. 1).

Como mencionamos, la adopción de las TIC han transformado la forma de comunicarnos, de buscar y transferir información en todos los ámbitos de la vida, y en la educación, como señalan los docentes, aumentan la flexibilidad para que estudiantes y profesores accedan al conocimiento, sin importar tiempo y barreras geográficas, y en la medida en que se han vuelto más sofisticadas, también se han vuelto más económicas y accesibles a través de diversos dispositivos electrónicos como el teléfono celular, que actualmente representa un acompañante ineludible de todo joven universitario.

\section{Necesidad de capacitación y actualización.}

Si bien la mayoría de los docentes entrevistados utilizan algún tipo de recurso tecnológico digital en su práctica docente, varios de los docentes se sienten limitados, incluso hay quienes reconocen no tener ningún conocimiento sobre ellos:

Pues muchas veces no entiendo mucho cómo se hacen, cómo se utiliza, o sea que siento que ya me quedé atrás ¿no?, cuando conocí los programas, pues no sabía nada, nada, lo reconozco, tengo que actualizarme (E3, profesora, Universidad CUDEC, p. 2).

(las TIC) supongo que podrían facilitar mil cosas para no andar de un lado para otro, pero si no sé usarlas ¿cómo podría estar del todo a favor? Si esto solo me complicaría la existencia porque tardaría más en realizar mis asuntos ya que no sabría cómo hacer algunas cosas, eso me llevaría más tiempo porque tendría que invertir tiempo en que alguien me enseñara, o para empezar en encontrar a ese alguien (E23, profesor, FES Zaragoza, p. 1).

La falta de competencias en el uso de las TIC que manifiestan los docentes se ha reportado por sobrados investigadores (Marín \& Romero, 2009; Moreno, 2009; Aráujo, \& Bermúdez, 2009; Guerra, González \& García, 2010; García, 2011; Echeverria, 2014; Raquimán, 2014; Araya \& Oreyana, 2018), ya sea que lo atribuyan a la falta de tiempo y espacios institucionales para capacitarse, por insuficiencia en la infraestructura, por escepticismo o resistencia a dejar sus prácticas de enseñanza al considerarlas eficaces. 
Se insiste así en la necesidad de una formación continua en el uso de la tecnologías, no solo mediante cursos aislados sino en forma transversal del currículo, es decir, la inclusión explícita de TIC en el currículo formal, como herramientas de apoyo a distintas materias y áreas del currículo y no como mera superposición, que permita a los docentes generar escenarios educativos que vayan más allá del uso del procesador de textos o de la tecnología disponible, y puedan satisfacer las necesidades formativas acorde a las estructuras cognitivas de los estudiantes y a lo que la sociedad del conocimiento exige, que presume como indispensable diseñar planes de formación del profesorado (Díaz Barriga y Moran, 2011).

\section{Problemas de infraestructura, conectividad y equipamiento.}

También de forma recurrente, los docentes revelaron enfrentar problemas técnicos y de reursos tecnológicos al emplear las TIC en su práctica educativa, ya sea porque la red inalámbrica falla o porque falta conexión en todos los espacios escolares, así como por insuficiencia o mal estado del equipo, por lo que es frecuente que utilicen sus propios recursos, prioritariamente desde sus hogares. Estos problemas son experimentados tanto por docentes de instituciones públicas como de privadas, aunque mayormente por los primeros:

Desafortunadamente en las aulas donde imparto clases ninguna cuenta con la infraestructura para hacer uso de las TIC (¿dónde las usa?) en casa, ya que, en la oficina los salones de clase o el cubículo no cuento con computadora para trabajar, ni internet (E17, profesora, FES Aragón, p.2).

No es suficiente (el equipo), si bien algunos salones tienen cañón, computadoras, algunos menos, pizarrones electrónicos, muchos de ellos tienen deficiencias, están descompuestos, no están calibrados, el cañón no funciona, no se ve entonces cómo? ... nosotros tenemos que subir nuestro equipo para trabajar y eso a la larga dificulta para que incorpores el uso de TIC en tu labor (E20, profesor, U. Autónoma del Edo. de Mex, Ecatepec, p. 2).

La falta de recursos y de infraestructura también es reportado de manera consistente en diversas investigaciones (Aráujo, \& Bermúdez, 2009; Díaz-Barriga \& Morán, 2011; Araya \& Oreyana, 2018), en cuyas instituciones indagadas, la infraestructura, conectividad y equipamiento son barreras a sortear diariamente. Al decir de los autores, las limitaciones en infraestrutura, unido a la falta de competencias digitales en los docentes, auguran un retraso importante de la aplicación de las TIC en el ámbito de las universidades que incidan y representen un cambio importante en las formas de apropiarse, producir y aplicar el conocimiento.

Si bien, en algunas universidades privadas si se cuenta con la infraestructura necesaria para que los docentes hagan uso de recursos digitales, y tienen el soporte pedagógico para su eficaz empleo, el problema en ocasiones es la falta de motivación y disposición de los docentes para su aprovechamiento:

La Ibero cuenta con el soporte tecnológico y constantemente brinda cursos de actualización para los docentes, pero la asistencia no es obligatoria por lo tanto muchos profesores no acuden (E24, profesora, U. Iberoamericana, p.1)

El acceso en las aulas al servicio de interconexión y a equipo de cómputo adecuado y actualizado con el softweare pertinente, resulta imprescindible para el uso óptimo de las TIC en los procesos educativos. Las instituciones tienen la responsabilidad de implementar los recursos necesarios si se quiere instaurar la innovación tecnológica en los recintos universitarios, pues 
aunque los docentes tengan la disponibilidad y apertura para formarse y actualizarse en las TIC, no podrán incorporarlas en su práctica docente. Si la escuela no considera importante el uso de las TIC en sus aulas, la infraestructura que posean va a ser escasa o nula (López, 2011).

Con todo y las deficiencias encontradas por algunos docentes de las instituciones privadas, éstas sortean mejor las condiciones de infraestructura y equipamiento para el uso de las TIC en la práctica educativa, en comparación de las instituciones públicas.

Sin embargo, no encontramos diferencias en las representaciones o significados que se atribuyen a las TIC por los docentes de ambas instituciones en lo que se refiere a la edad, sexo, antigüedad en la docencia, turno, o materias impartidas de los docentes. Por lo que estas variables no parecen tener un impacto significativo y diferenciado en la diversidad de representaciones develadas en los ejes analizados.

\section{Discusión}

La investigación realizada, si bien no es concluyente, nos permitió un acercamiento de tipo descriptivo e interpretativo a las formas en que docentes que enseñan contenidos de psicología representan las TIC, y a las formas en que las incorporan en su práctica docente. En general, existe una visión y empleo de las TIC de tipo instrumental y pragmático entre los docentes, que aún y cuando nuestra intención no fue generalizar los hallazgos obtenidos, éstos coinciden de manera muy cercana con los encontrados por otros investigadores (Moreno, 2009; Díaz-Barriga y Morán, 2011; Araya y Orellana, 2018).

Los docentes de nuestra investigación atribuyen a las TIC usos potenciales y valiosos para la educación, y las representan como herramientas innovadoras e indispensables para la práctica educativa, sin embargo, las emplean solo para la búsqueda de información y como canales de comunicación y distribución de materiales de aprendizaje, o para la búsqueda de apoyos visuales que permitan focalizar o mantener la atención de los estudiantes, y solo en casos excepcionales, las emplean como estrategia pedagógica que permita la apropiación y construcción o reconstrucción del conocimiento.

Indudablemente los apoyos audiovisuales y el acceso rápido a todo tipo de información representan un soporte tecnológico facilitador para inducir las actividades de aprendizaje, pero no garantizan por si mismos el aprendizaje significativo, crítico y propositivo que la educación debiera promover, máximo en la educación superior, y en particular en carreras como la psicología en la que el estudiante se enfrenta a infinidad de conocimientos, paradigmas y formas de investigar e intervenir en sus diferentes áreas de conocimiento.

Es importante distinguir aquí que el uso de internet, obtener y distribuir información, utilizar videos, esquemas, presentaciones como apoyo a la enseñanza, el empleo de las redes sociales para comunicarse con los estudiantes, enviar instrucciones o distribuir materiales académicos, es decir, poseer "competencias digitales" es un asunto, y otro es la intervención pedagógica y didáctica del docente que incite en los estudiantes la comprensión de la información, la integren a sus estructuras cognitivas y la usen en otros contextos para solucionar problemas. En la sociedad del conocimiento y en un mundo globalizado, esto es lo que se demanda de nuestros egresados universitarios.

La forma en que los docentes han incorporado las TIC en su práctica docente, nos remite a recordar el impacto que en los años sesenta y setentas del siglo pasado tuvieron las aportaciones de la Tecnología Educacional en los sistemas escolares. La Tecnología Educacional recuperó los medios audiovisuales de las comunicaciones de esa época (TV, radio, transparencias, proyector 
de trasparencias, retroproyectores de acetatos, rotafolios, etc.) y los principios de la psicología conductual con énfasis en la definición de objetivos y la enseñanza programada, para elevar el desempeño académico sin que se cambiaran las condiciones del aprendizaje receptivo que promueve la enseñanza tradicional (véase Chadwick, 1987). Al respecto, es muy elocuente el argumento que una de las docentes entrevistadas manifestó "muchas veces utilizamos diapositivas en lugar del bond, o sea, es lo mismo, nada más que uno es proyectado y el otro es en papel" (E8, profesora, FES Iztacala y UPN).

En este sentido, la forma en que los docentes que enseñan contenidos de psicología adoptan las TIC en su práctica, difícilmente les permitirá superar los problemas de la enseñanza enciclopedista de la psicología, o rebasar el énfasis puesto en los aprendizajes teóricos y conceptuales. En otras palabras, no basta la innovación tecnológica por si misma o su disponibilidad para elevar la calidad educativa, menos para cambiar tradiciones de enseñanza en una disciplina en particular. Se necesita ir más allá que la mera utilización de la información, software o recursos digitales disponibles en la WEB para promover la profundización y construcción del conocimiento.

Es indispensable que los docentes rebasen el uso instrumental de las TIC, desarrollen habilidades y apliquen su creatividad para diseñar ambientes de aprendizaje que promuevan el desarrollo de habilidades profesionales y el pensamiento complejo en los estudiantes. Esto es, se requiere que trasciendan lo que se considera la Educación/Web 1.0 que contempla simples herramientas para eficientar la enseñanza (tecnología de acceso para la obtención de datos e información), en la que los estudiantes obtienen información de manera pasiva, con pocos espacios para la interacción y colaboración de los usuarios; y se trasladen a la Web 2.0, caracterizada por fomentar el diseño de ambientes de enseñanza-aprendizaje interactivas. Entre las alternativas de formación de los docentes que se plantean, se propone la adquisición de competencias para la utilización de otras herramientas como son las ofrecidas por la Web 2.0 (herramientas colaborativas online, blogs, foros y marcadores) (Guerra, González y García, 2010).

Sin embargo, esta formación docente reclama, como analizamos, rebasar los cursos aislados o superpuestos al currículo. Para ello, es apremiante primero, promover que los docentes analicen sus representaciones y los significados que asignan a la innovación y a la calidad educativa. Posteriormente, es importante que reflexionen y asuman que la incorporación de las TIC requiere de una actividad intencional y planeada para la creación de condiciones de enseñanza que realmente impliquen una innovación pedagógica (Fullan y Smith, 1999, cit. por Salinas, 2004).

La incorporación de las TIC en la práctica docente representará realmente una innovación pedagógica solo si mediante éstas se diseñan escenarios educativos que promuevan intercambios comunicativos entre docentes y estudiantes, en los que se pongan en juego procesos cognitivos, reflexivos y creativos, que permitan a los estudiantes una participación más activa en su aprendizaje, que faculte los aprendizajes disciplinarios de la psicología, pero también un nivel de conocimientos y saberes más prácticos y reflexivos para la trasformación de las prácticas profesionales.

\section{Referencias}

Abdulzaman, K. A. \& Azizah, A. R. (2013). Facebook in higher education: students' use and perceptions. Advances in information sciences and service sciences, 5(15), 32-42. 
Recuperado

https://pdfs.semanticscholar.org/f5c0/dbe0b6226a2450fe89356e3ca6fd02217dd6.pdf

Abric, J. C (2001). Las representaciones sociales: aspectos teóricos. En Abric, J. C. (Dir.), Prácticas sociales y representaciones (pp.11-32). México: Coyoacán.

Almeida, E., Guarneros, N., Limón, A. \& Román, S. (1989). Presente y futuro del trabajo profesional del psicólogo en México. En Urbina, J. (Comp.), El psicólogo: formación, ejercicio profesional y prospectiva (pp.787-791). México: UNAM.

Aráujo, D., \& Bermúdez, J. (2009). Limitaciones de las tecnologías de información y comunicación en la educación universitaria. Horizontes Educacionales, 14(1), 9-24. Recuperado de https://www.redalyc.org/pdf/979/97912444001.pdf

Araya, V. \& Orellana, X. (2018). Representaciones de docentes universitarios respecto de las TIC en la acción práctica: Algunas claves para el diseño de constancias formativas. Revista de Estudios y Experiencias en Educación, Número Especial 2(3), 45-58. Recuperado de http://www.rexe.cl/ojournal/index.php/rexe/article/view/547

Castañeda, S. (1999). Algunos referentes para un análisis crítico para la preparación profesional del Licenciado en Psicología en México. Una experiencia colegiada. En Vázquez, G. \& Martínez, J. (Eds.), La formación del Psicólogo en México (pp. 25-41). México: Universidad Latinoamericana.

Castaño, D. (1989). Prospectiva del ejercicio profesional de la psicología en México. En Urbina, J. (comp.), El psicólogo: formación, ejercicio profesional y prospectiva (pp. 779-785). México: UNAM.

Chadwick, C. (1987). Tecnología Educacional para el docente (2a. Ed.). Barceloa: Paidós.

Coll, C. (2008). Aprender y enseñar con las TIC: expectativas, realidad y potencialidades. En Carneiro, R., Toscano, J. C. y Díaz, T. (comp.). Los desafíos de las tic para el cambio educativo (pp. 113-128). Madrid: OEI-Santillana, Fundación Santillana.

Covarrubias, P. (2003). Currículum, disciplina y profesión, desde la perspectiva de los académicos de Psicología Iztacala. Tesis de doctorado en Pedagogía. México: Facultad de Filosofía y Letras- UNAM.

Covarrubias, P. (2017). Representaciones de profesores universitarios acerca de las Tecnologías de la Información y Comunicación (TIC) en el currículo y su incorporación a la práctica docente. Ponencia presentada en el XIV Congreso Nacional de Investigación Educativa del COMIE. Aportes y desafíos de la investigación educativa para la transformación y la justicia social, San Luis Potosí, Mex. 20-24 noviembre. Recuperado de http://www.comie.org.mx/congreso/memoriaelectronica/v14/doc/1568.pdf

Covarrubias, P. \& Casarini, M. (2013). Los actores del currículo en México: Un campo de conocimiento en constitución. En Díaz Barriga, A. (Coord.). La investigación curricular en México. La primera década del siglo XXI (2002-2012) pp. (197-262). Colección Estados del Conocimiento: COMIE-ANUIES.

Covarrubias, P. \& E. Camarena, O. (2010). Construcción del conocimiento e identidad profesional. México: UNAM-Castellanos Editores.

Díaz-Barriga, F. \& Morán, H. (2011). Usos y niveles de apropiación de las TIC con fines de enseñanza en profesores universitarios de Psicología Educativa. En Díaz Barriga, F., 
Hernández, G. \& Rigo, M. C. (Eds.), Experiencias educativas con recursos digitales. Prácticas de uso y diseño tecnopedagógico (49-68). México: Facultad de PsicologíaUNAM.

Echeverria, A. C. (2014). Uso de las TIC en la docencia universitaria: opinión del profesorado de educación especial. Revista Electrónica "Actualidades investigativas en Educación”, 4(3), 1-24. Recuperado de https://www.scielo.sa.cr/pdf/aie/v14n3/a12v14n3.pdf

Erickson, F. (1989). Métodos cualitativos de investigación sobre la enseñanza. En Wittrock, M. c. La investigación de la enseñanza, 2. Barcelona: Paidós.

García-Valcácer, A., Hernández, A. \& Recamán, A. (2012). La metodología del aprendizaje colaborativo a través de las TIC: una aproximación a las opiniones de profesores y alumnos. Revista Complutense de Educación, 23(1), 161-188. Recuperado de https://revistas.ucm.es/index.php/RCED/article/view/39108/37721

García, A. (2011). Concepciones sobre uso de las TIC del docente universitario en la práctica pedagógica. Anuario Electrónico de Estudios en Comunicación Social "Disertaciones", 4 (1), Artículo 9 . Recuperado de https://revistas.urosario.edu.co/index.php/disertaciones/article/view/3906

Gimeno, S. (1995). El currículum: una reflexión sobre la práctica (5ª ed.). Madrid: Morata

Gimeno, S. \& Pérez Gómez, A. (1989). (Comp.). La enseñanza, su teoría y su práctica. Madrid: Akal-Universitaria.

Guerra, S., González, N. \& García, R. (2010). Utilización de las TIC por el profesorado universitario como recurso didáctico. Revista Cientifica Educomunicación, 35(18), 141148. DOI:10.3916C35-2010-03-07.

Hammersley, M. \& Atkinson, P. (1994). Etnografía. Métodos de Investigación. México: Paidós.

Harrsch, C. (1998). Identidad del Psicólogo. México: Pearson Educación.

Herrera, A. (1993). La formación profesional del psicólogo. Perfiles Educativos, (59), 31-44. Recuperado de http://www.iisue.unam.mx/perfiles/perfiles_articulo.php?clave=1993-59$31-44$

Lara, L. (1989). La profesión de psicólogo: un análisis de sus antecedentes, situación actual y futura. En Urbina, J. (Comp.), El psicólogo: formación, ejercicio profesional y prospectiva (769-778). México: UNAM.

Marín, D. V. \& Romero, L. M. (2009). La formación del docente universitaria a través de las TICS. Revista de Medios y Educación, (35), 97-103. Recuperado de: https://www.redalyc.org/pdf/368/36812381008.pdf

Monereo, C. \& Pozo, J. I. (2008). El alumno en entornos virtuales. Condiciones, perfiles y competencias. En Coll, C. y Monereo, C. (Eds.) Psicología de la Educación Virtual (pp. 110-131). Madrid: Morata.

Moreno, T. J. (2009). Diagnóstico de los usos e incorporación de las TIC en un escenario univesitario. En Díaz Barriga, F., Hernández, G. \& Rigo M. Aprender y enseñar con TIC en Educación superior: contribuciones del socioconstructivismo (pp. 207-204). México: Facultad de Psicología, UNAM.

Moscovici, S. (1986). Psicología social II. Pensamiento y vida social. Psicología social y problemas sociales. Barcelona: Paidós. 
Noriega, J. A. \& Gutiérrez, C. (1995). Introducción a la epistemología para psicólogos. México: Plaza y Valdez.

OCDE (2003). ICT and Economic Growth. Paris: OCDE Publication Service. Recuperado de http://www.cla.org.pt/docs/OCDE_TIC.PDF

PÉREZ, C. I. (2011). "El papel del profesor y el uso de TIC como herramienta de apoyo". En F. Díaz Barriga, G. Hernández y M. C. Rigo, (Eds.), Experiencias educativas con recursos digitales. Prácticas de uso y diseño tecnopedagógico (213-234). México: Facultad de Psicología UNAM.

Preciado, H. \& Rojas, L. A. (1989). Notas sobre la enseñanza de la psicología en México: estado actual y perspectivas de desarrollo. Revista de la Educación Superior, 18(72), 57-72. Recuperado de http://publicaciones.anuies.mx/pdfs/revista/Revista72_S1A5ES.pdf

Raquimán, P. 2014). Representaciones sobre el cambio en el uso de las TIC. Relatos de vida de profesores. Revista Iberoamericana de Educación, (65), 75-90. DOI: https://doi.org/10.35362/rie650394

Riascos-Erazo, S., Quintero-Calvache, D. \& Ávila-Fajardo, G. (2009). Las TIC en el aula: percepciones de los profesores universitarios. Educación y Educadores, 12(2), 133-157. Recuperado de https://www.redalyc.org/pdf/834/83412235008.pdf

Romero, V. \& Hernández, G. (2011). Usos y apropiación de las TIC en estudiantes universitarios. En F. Díaz Barriga, G. Hernández \& Rigo, M. C. (Eds.), Experiencias educativas con recursos digitales. Prácticas de uso y diseño tecnopedagógico (27-48). México: Facultad de Psicología UNAM.

Salinas, J. (2004). Innovación docente y uso de las TIC en la enseñanza universitaria. Revista Universidad y Sociedad del Conocimiento, 1(1), 1-16. Recuperado de https://www.redalyc.org/pdf/780/78011256001.pdf

Salinas, S. A. (2008). Softweare para Trabajo Colaborativo y Bibliotecas. Monte Grande: Instituro Superior de Formación Docente y Técnica $\mathrm{N}^{\circ}$. 35. Recuperado de https://core.ac.uk/download/pdf/11886903.pdf

Taylor, S. J. \& Bodgan, R. (1990). Introducción a los métodos cualitativos de investigación. Barcelona: Paidós.

UNESCO (2008). Estándares de Competencias en TIC para docentes. París: Organización de las Naciones Unidas para la Educación, la Ciencia y la Cultura. Recuperado de http://www.eduteka.org/EstandaresDocentesUnesco.php

Young, E. (1981). A study of teacher epistemologies. The Australian Journal of Education, 25(2), 194-208. Recuperado de https://doi.org/10.1177/000494418102500208.

Zanatta, E. \& Yurén, T. (2012). La formación profesional del psicólogo en México: trayecto de la construcción de su identidad disciplinar. Enseñanza e Investigación en Psicología, 17(1), 151-170. Recuperado de http://www.redalyc.org/pdf/292/29223246010.pdf 